\title{
Colonization of Supplemented
} Bifidobacterium breve M-16V in Low Birth Weight Infants and Its Effects on Their Gut Microbiota Weeks Post-administration

\author{
Ayako Horigome ${ }^{1 * t}$, Ken Hisata ${ }^{2 \dagger}$, Toshitaka Odamaki ${ }^{1}$, Noriyuki Iwabuchi ${ }^{3}$, \\ Jin-zhong Xiao ${ }^{1}$ and Toshiaki Shimizu ${ }^{2}$
}

${ }^{1}$ Next Generation Science Institute, Morinaga Milk Industry Co., Ltd., Kanagawa, Japan, ${ }^{2}$ Department of Pediatrics, Juntendo University Faculty of Medicine, Tokyo, Japan, ${ }^{3}$ Food Ingredients and Technology Institute, Morinaga Milk Industry Co., Ltd., Kanagawa, Japan

OPEN ACCESS

Edited by:

María Esteban-Torres, University College Cork, Ireland

Reviewed by:

Angelica Thomaz Vieira, Federal University of Minas Gerais,

Brazi

Marie-Jose Butel, Université de Paris, France

*Correspondence: Ayako Horigome a-horigome@morinagamilk.co.jp

${ }^{\dagger}$ These authors have contributed equally to this work

Specialty section: This article was submitted to Microbial Symbioses,

a section of the journal Frontiers in Microbiology

Received: 25 September 2020 Accepted: 17 March 2021 Published: 07 April 2021

Citation:

Horigome A, Hisata K, Odamaki T, Iwabuchi N, Xiao J-z and Shimizu T (2021) Colonization of Supplemented Bifidobacterium breve M-16V in Low Birth Weight Infants and lts Effects on Their Gut Microbiota Weeks Post-administration Front. Microbiol. 12:610080. doi: 10.3389/fmicb.2021.610080
The colonization and persistence of probiotics introduced into the adult human gut appears to be limited. It is uncertain, however, whether probiotics can successfully colonize the intestinal tracts of full-term and premature infants. In this study, we investigated the colonization and the effect of oral supplementation with Bifidobacterium breve M-16V on the gut microbiota of low birth weight (LBW) infants. A total of 22 LBW infants (12 infants in the M-16V group and 10 infants in the control group) were enrolled. B. breve M-16V was administrated to LBW infants in the M-16V group from birth until hospital discharge. Fecal samples were collected from each subject at weeks (3.7-9.3 weeks in the M-16V group and 2.1-6.1 weeks in the control group) after discharge. qPCR analysis showed that the administrated strain was detected in $83.3 \%$ of fecal samples in the M-16V group (at $\log _{10} 8.33 \pm 0.99$ cell numbers per gram of wet feces), suggesting that this strain colonized most of the infants beyond several weeks post-administration. Fecal microbiota analysis by $16 \mathrm{~S}$ rRNA gene sequencing showed that the abundance of Actinobacteria was significantly higher $(P<0.01)$, whereas that of Proteobacteria was significantly lower $(P<0.001)$ in the $\mathrm{M}-16 \mathrm{~V}$ group as compared with the control group. Notably, the levels of the administrated strain and indigenous Bifidobacterium bacteria were both significantly higher in the M-16V group than in the control group. Our findings suggest that oral administration of $B$. breve $\mathrm{M}-16 \mathrm{~V}$ led to engraftment for at least several weeks post-administration and we observed a potential overall improvement in microbiota formation in the LBW infants' guts.

Keywords: low birth weight infants, gut microbiota, Bifidobacterium breve M-16V, probiotics, colonization

\section{INTRODUCTION}

The gut microbiota in infancy plays many important roles underpinning healthy development and thereby impacts future health. Recent studies have indicated that there is a link between infant gut dysbiosis and an increased risk of developing acute and long-term inflammatory diseases in later life such as asthma (Arrieta et al., 2015, 2018; Zimmermann et al., 2019), type 1 diabetes (Kostic et al., 2015) and obesity (Cox et al., 2014). Low birth weight (LBW) preterm infants have important 
differences in the composition of their intestinal microbiota when compared with full-term infants (Henderickx et al., 2019). These differences are related to immaturely-developed guts and receiving antibiotic treatments, as well as the neonatal intensive care hospital environment itself, which limits a preterm infant's contact with commensal bacteria (Henderickx et al., 2019). The typical gut microbiota of preterm infants is characterized by the presence of potentially pathogenic bacteria commonly found in the hospital environment, such as Klebsiella, Escherichia, Staphylococcus, and Enterococcus (Patel et al., 2016; Stewart et al., 2016). Another characteristic is the low abundance of Bifidobacterium (Dalby and Hall, 2020), which is the most common genus in the normal infant gut and is thought to play pivotal roles in maintaining infant health (Leahy et al., 2005; Di Gioia et al., 2014). Combined with the underdeveloped gut and immune system in premature infants, gut dysbiosis increases their susceptibility to conditions such as sepsis and necrotizing enterocolitis (NEC) (Neu and Pammi, 2017), the latter of which is the most common and lethal gastrointestinal emergency for them. Therefore, early intervention to improve gut dysbiosis is essential for infants, especially premature ones.

Administration of probiotic Bifidobacterium strains is one potential approach for establishing normal gut microbiota in premature infants. Previous research studies have shown that supplementation with Bifidobacterium strains results in higher numbers of Bifidobacterium and the lower counts of Enterobacteriaceae in premature infants (Mohan et al., 2006; Ishizeki et al., 2013). Bifidobacterium breve M-16V is a probiotic strain originating from a healthy infant and has been incorporated into several products including infant formula (Wong et al., 2019). It has received GRAS status for foods including infant formula from the US Food and Drug Administration (GRAS No. 453-455). This strain has been shown to have gut microbiota modulating potential in infants and can protect against preterm- and infant-related diseases (Wong et al., 2019), although adequately powered, preferably cluster randomized controlled trials are needed to confirm these findings (Athalye-Jape et al., 2018). For example, a randomized, double-blind, placebo-controlled trial showed that B. breve M$16 \mathrm{~V}$ supplementation for three weeks resulted in a significantly higher abundance of $B$. breve in the feces of preterm infants, unlike the placebo control group that had $B$. breve counts below the detection level (Patole et al., 2014). It was also reported that daily supplementation with $B$. breve $\mathrm{M}-16 \mathrm{~V}$ decreased the incidence of NEC in very low birth weight neonates with a birth gestational age less than 34 weeks (Patole et al., 2016). The study was a retrospective cohort study involving 835 preterm neonates as historical controls and 920 preterm neonates receiving this strain. An experimental rat NEC model supported the preventive effect of $B$. breve $\mathrm{M}-16 \mathrm{~V}$ administration on NEC and revealed that the mechanism involved modulation of Toll-like receptor expression and inflammatory response suppression (Satoh et al., 2016). To date, $B$. breve $\mathrm{M}-16 \mathrm{~V}$ has been used to reduce the risk of preterm birth complications with LBW infants in more than 120 neonatal intensive care units (NICUs) in affiliated hospitals in Japan, Australia, New Zealand and Singapore (Umezaki et al., 2010; Patole et al., 2014, 2016; Athalye-Jape et al., 2018). As mentioned above, although live cells of $B$. breve $\mathrm{M}-16 \mathrm{~V}$ have been confirmed to have effects on gut microbiota and infant health, little information exists about colonization of this strain in the gut and whether colonization following probiotic supplementation has a prolonged effect on gut microbiota.

Therefore, in the present study, we administered B. breve M$16 \mathrm{~V}$ to LBW infants (gestation $\leq 37$ weeks) admitted to NICU from birth to hospital discharge and followed up these patients for several weeks after discharge. We observed that $B$. breve $\mathrm{M}-16 \mathrm{~V}$ colonized in the gut and contributed to a potential improvement of the gut microbiota composition in the LBW infants at least for several weeks post-administration.

\section{MATERIALS AND METHODS}

\section{Subjects and Sample Collection}

This study was reviewed and approved by the Ethics Committee of Juntendo University Hospital, Japan, and written informed parental consent was obtained. The outline of study schedule for each subject is shown in Figure 1. A total of 22 infants with gestational ages $\leq 37$ weeks and birth weights $<2,000$ gram who were admitted to the NICU of the Juntendo University Urayasu Hospital (control group, $n=10$ ) or the Juntendo University Hospital (M-16V group, $n=12$ ) from March 2012 to February 2017 were enrolled. After birth, infants in the M-16V group were daily administrated a freeze-dried preparation of $B$. breve M$16 \mathrm{~V}$ (dose, $1 \times 10^{9} \mathrm{CFU}$ dissolved in $4 \mathrm{~mL}$ of sterile water) just before feeding. The probiotic administration was continued until hospital discharge. Infants in the control group were not administrated any probiotic supplement. At 2-9 weeks after hospital discharge, fresh fecal samples were collected from each subject's diaper to a tube. The fecal samples collected were stored below $-18^{\circ} \mathrm{C}$ until delivery to the laboratory. Immediately upon receipt, the fecal samples were stored at $-80^{\circ} \mathrm{C}$ until further analysis. Details of the study schedule for each subject is shown in Supplementary Table 1.

\section{Fecal DNA Preparation and Microbiota Analysis}

DNA was extracted from the fecal samples as previously described (Sugahara et al., 2015). Purified DNA was suspended in 2,000 $\mu$ l of Tris-EDTA buffer ( $\mathrm{pH} 8.0$ ).

PCR amplification and DNA sequencing of the V3-V4 region of the bacterial 16S rRNA gene was performed on the Illumina MiSeq instrument (Illumina, San Diego, CA, United States) as previously described (Odamaki et al., 2016). After removing the sequences consistent with the data from the Genome Reference Consortium human build 38 (GRCh38) and the phiX reads from the raw Illumina paired-end reads, the sequences were analyzed using the QIIME2 software package (version 2017.10) ${ }^{1}$. Potential chimeric sequences were removed using DADA2 (Callahan et al., 2016), and 30 and 90 bases were trimmed from the $3^{\prime}$ region of the forward and the reverse reads, respectively. Taxonomical classification was performed using the Naive Bayes classifier

\footnotetext{
${ }^{1}$ https://qiime2.org/
} 


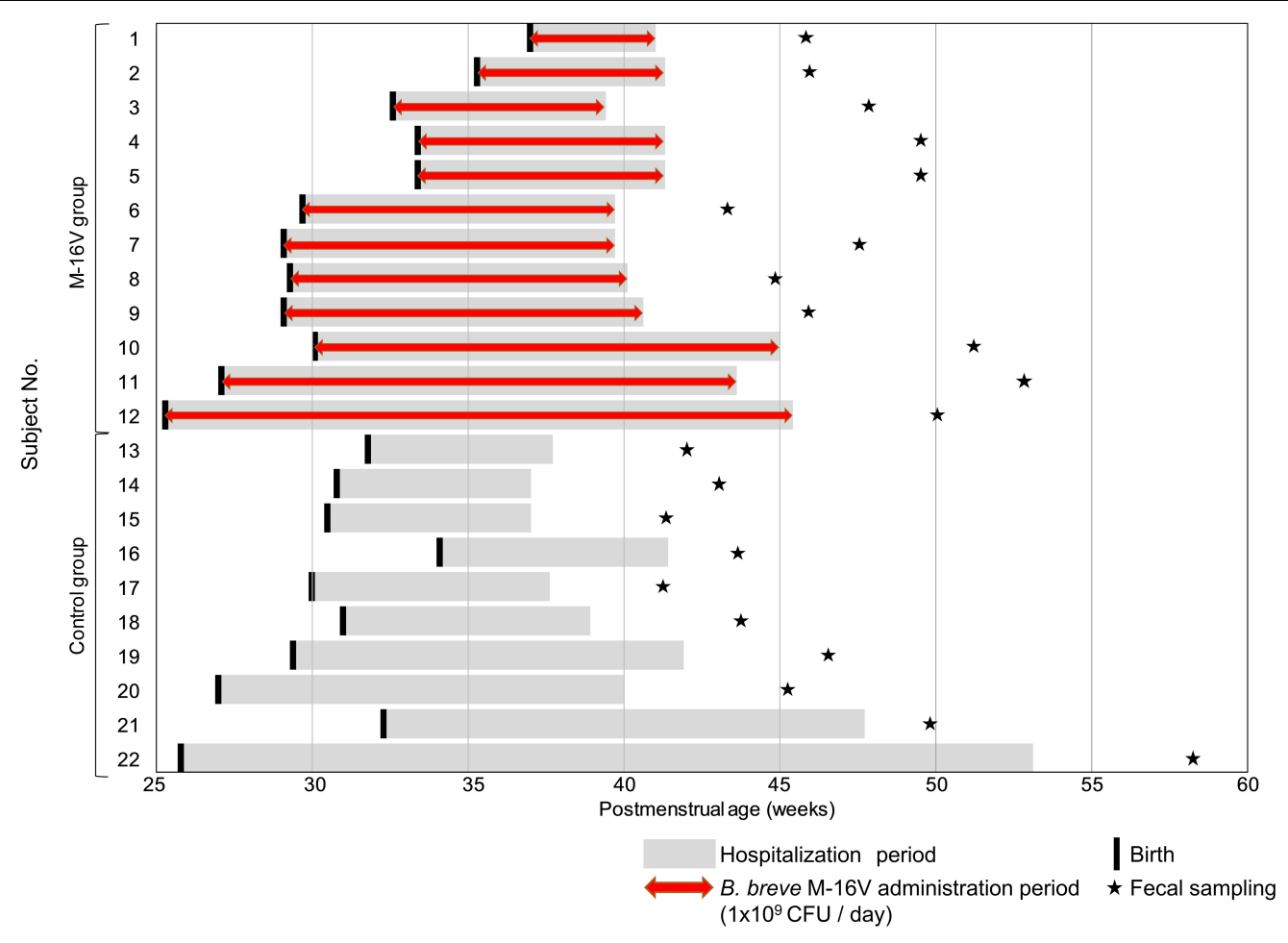

FIGURE 1 | Outline of study schedule for each subject across postmenstrual age.

trained on the Greengenes13.8 dataset with a 99\% sequence similarity threshold for full-length Operational Taxonomic Units.

\section{Quantitative PCR (qPCR)}

The fecal DNAs as described above were applied for qPCR which was performed on the ABI PRISM 7500 Fast RealTime PCR system (Thermo Fisher Scientific K.K., Uppsala, Sweden) with SYBR Premix Ex Taq (TaKaRa Bio, Shiga, Japan) to quantitate the Bifidobacterium species and strains. The primer sets used are shown in Supplementary Table 2. The primers specific for Bifidobacterium longum, Bifidobacterium catenulatum, and Bifidobacterium adolescentis groups have been described previously (Matsuki et al., 1998, 2004). The B. breve M-16V-specific primer set was designed using Primer 3 software (v.0.4.0) (Untergasser et al., 2012) after identifying unique regions in this strain by multiple sequences alignments of the complete genome sequences of $B$. breve $\mathrm{M}-16 \mathrm{~V}$ and publically available genomes of $B$. breve strains. We confirmed the specificity of this specific primer set using other 37 B. breve strains in Morinaga Culture Collection.

PCR amplification was performed using the program previously described (Kato et al., 2017) with the exception of $B$. breve $\mathrm{M}-16 \mathrm{~V}$, whose detection consisted of an amplification program of one cycle at $95^{\circ} \mathrm{C}$ for $20 \mathrm{~s}, 40$ cycles at $95^{\circ} \mathrm{C}$ for $3 \mathrm{~s}$ and $60^{\circ} \mathrm{C}$ for $30 \mathrm{~s}$, and one final cycle at $95^{\circ} \mathrm{C}$ for $15 \mathrm{~s}$. The following Bifidobacterium strains were used as the standards

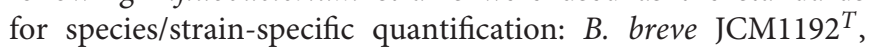
B. longum subsp. longum JCM1217 ${ }^{T}$, Bifidobacterium bifidum
JCM1255 ${ }^{T}$, Bifidobacterium pseudocatenulatum JCM1200 ${ }^{T}$, B. adolescentis $\mathrm{JCM} 1275^{T}$, and $B$. breve M-16V.

\section{Statistical Analysis}

Statistical analyses were performed using EZR software ver. 1.50 (Kanda, 2013) or R software ver. 3.6.0. Intergroup differences were analyzed using the unpaired Student's $t$-test or Welch's $t$-test, and the Mann-Whitney $U$-test, for parametric and nonparametric data, respectively. Fisher's exact test or $\chi^{2}$ test was conducted for categorical data. The cell number calculations for Bifidobacterium species or strains were substituted by $\log _{10} 6$ per gram of wet feces for samples that fell below the detection limits. Differences in the gut microbiota profiles between the control and $\mathrm{M}-16 \mathrm{~V}$ groups were analyzed by principal coordinate analysis (PCoA). A permutational multivariate analysis of variance (PERMANOVA) test for UniFrac distances was used for multivariate analysis to test the variation in microbiota composition explained by each factor. Associations between relative abundance of Actinobacteria or Proteobacteria and subject's characteristics were assessed by Spearman's rank correlation test. For all statements, $P<0.05$ were considered to be statistically significant.

\section{Data Availability}

DNA sequences corresponding to the $16 \mathrm{~S}$ rRNA gene data have been deposited in the DNA Data Bank of Japan (DDBJ) under accession number DRA010463. 


\section{RESULTS}

\section{General Characteristics of the Subjects}

Altogether, 22 infants (10 in the control group and 12 in the M-16V group) were enrolled in this study. Table 1 shows the characteristics of the subjects. There was no significant difference in the maternal features between the groups. As neonatal features, infants were matched for gender, gestational age and breast-feeding rate, and the hospitalization period/supplementation duration, postnatal age, and corrected age at the fecal sampling were comparable between the two groups. However, birth weight and discharge weight were significantly higher in the $\mathrm{M}-16 \mathrm{~V}$ group than in the control group. The period from hospital discharge to fecal sampling was significantly longer in the M-16V group than in the control group.

TABLE 1 | Characteristics of the subjects.

\begin{tabular}{|c|c|c|c|}
\hline & $\begin{array}{c}\text { M-16V group } \\
(n=12)\end{array}$ & $\begin{array}{l}\text { Control group } \\
(n=10)\end{array}$ & $P$-value \\
\hline \multicolumn{4}{|l|}{ Maternal features } \\
\hline Age (years) & $34.0 \pm 4.0$ & $31.3 \pm 3.3$ & 0.100 \\
\hline C-section (n, \%) & $8(66.7)$ & $9(90)$ & 0.323 \\
\hline $\begin{array}{l}\text { Antibiotics during labor } \\
(n, \%)\end{array}$ & $10(83.3)$ & $9(90)$ & 1.000 \\
\hline \multicolumn{4}{|l|}{ GBS test $(n, \%)$} \\
\hline Positive & $1(8.3)$ & $1(10)$ & 1.000 \\
\hline Negative & $8(66.7)$ & $7(70)$ & \\
\hline Not done & $3(25)$ & $2(20)$ & \\
\hline \multicolumn{4}{|l|}{ Neonatal features } \\
\hline Gestational age (weeks) & $31.0 \pm 3.4$ & $30.3 \pm 2.4$ & 0.613 \\
\hline Birth weight (g) & $1350.5 \pm 250.1$ & $1096.3 \pm 254.7$ & $0.029^{*}$ \\
\hline Discharged weight (g) & $3430.3 \pm 926.1$ & $2630.0 \pm 451.1$ & $0.017^{\star}$ \\
\hline Male $(n, \%)$ & 7 (58.3) & $3(30)$ & 0.369 \\
\hline Chronic lung disease & $1(8.3)$ & $1(10)$ & 1.000 \\
\hline $\begin{array}{l}\text { Antibiotics exposure } \\
(n, \%)\end{array}$ & $5(41.7)$ & $3(30)$ & 0.675 \\
\hline \multicolumn{4}{|l|}{ Treatment for PDA } \\
\hline Clipping $(n, \%)$ & $1(8.3)$ & $1(10)$ & 0.814 \\
\hline Indomethacin $(n, \%)$ & $3(25)$ & $4(40)$ & \\
\hline $\begin{array}{l}\text { Supplementation } \\
\text { duration/hospitalization } \\
\text { period (weeks) }\end{array}$ & $10.6 \pm 4.6$ & $10.9 \pm 6.6$ & 0.887 \\
\hline Breast-feeding $(\%)^{\dagger}$ & $54.2 \pm 39.6$ & $45.0 \pm 23.0$ & 0.526 \\
\hline $\begin{array}{l}\text { Fecal sampling (weeks } \\
\text { after discharge) }\end{array}$ & $6.4 \pm 1.9$ & $4.3 \pm 1.3$ & $0.009^{\star \star}$ \\
\hline $\begin{array}{l}\text { Postnatal age at the } \\
\text { fecal sampling (weeks) }\end{array}$ & $17.0 \pm 5.0$ & $15.3 \pm 6.8$ & 0.509 \\
\hline $\begin{array}{l}\text { Corrected age at the } \\
\text { fecal sampling (weeks) }\end{array}$ & $7.9 \pm 2.8$ & $5.5 \pm 5.2$ & 0.184 \\
\hline
\end{tabular}

Measured variable data are expressed as the mean $\pm S D$. Intergroup differences were analyzed using the $x^{2}$-test or Fisher's exact test for categorical data and the unpaired Student's $t$-test or Welch's $t$-test for measured variables. ${ }^{*} P<0.05$; ${ }^{* *} P<0.01$.

†Percentage of breast milk among the total nutrition (breast milk + infant formula), as surveyed by questionnaire. GBS, Group B Streptococcus; PDA, patent ductus arteriosus.

\section{Effect of B. breve M-16V Administration on the Gut Microbiota of the Infants}

To evaluate the effect of administering probiotics during early life on the composition of fecal microbiota, we collected fecal samples 3-9 weeks after their administration. No significant difference in the alpha diversity of the microbiota was observed between the groups (Supplementary Table 3). PCoA of the fecal microbiota based on the weighted UniFrac distance indicated that $B$. breve $\mathrm{M}-16 \mathrm{~V}$ administration had an impact on the composition of the fecal microbiota (Figure 2). PERMANOVA testing revealed a significant difference in the gut microbiota profiles of the $\mathrm{M}-16 \mathrm{~V}$ and control groups. As shown in Table 2 and Figure 3, the dominant phylum identified in the $\mathrm{M}-16 \mathrm{~V}$ group was Actinobacteria (74\%), followed by Firmicutes (19.9\%). In contrast, the gut microbiota from the control group showed a higher abundance of Proteobacteria (22.7\%) than that of the M-16V group (3\%). The relative abundance of Actinobacteria was significantly higher, whereas that of Proteobacteria was significantly lower in the M-16V group than in the control group. At the genus level, the relative abundances of Bifidobacterium and Enterococcus were significantly higher, whereas those of Rothia, Lactococcus, and Klebsiella were significantly lower in the M-16V group than in the control group.

TABLE 2 | Fecal microbiota composition

\begin{tabular}{|c|c|c|c|}
\hline & \multicolumn{2}{|c|}{ Median (IQRs) \% } & \multirow[t]{2}{*}{$P$-valu } \\
\hline & $\begin{array}{c}\text { M-16V group } \\
(n=12)\end{array}$ & $\begin{array}{c}\text { Control group } \\
\quad(n=10)\end{array}$ & \\
\hline \multicolumn{4}{|l|}{ Phylum } \\
\hline Actinobacteria & $74.0(61.9-79.8)$ & $33.3(3.0-64.9)$ & $0.009^{\star \star}$ \\
\hline Bacteroidetes & $0.0(0.0-0.3)$ & $0.2(0.1-0.3)$ & 0.132 \\
\hline Firmicutes & $19.9(17.5-30.7)$ & $31.9(15.7-48.6)$ & 0.628 \\
\hline Proteobacteria & $3.0(0.6-5.4)$ & $22.7(14-46.7)$ & $0.000^{* \star}$ \\
\hline \multicolumn{4}{|l|}{ Genus } \\
\hline Bifidobacterium & $73.44(60.96-78.87)$ & $32.19(2.56-64.28)$ & $0.011^{*}$ \\
\hline Clostridiaceae |g_ & $0.08(0.00-0.37)$ & $0.30(0.16-0.34)$ & 0.366 \\
\hline Clostridiales |___ & $0.29(0.04-0.89)$ & $0.15(0.05-0.25)$ & 0.531 \\
\hline Clostridium & $0.13(0.00-0.64)$ & $0.29(0.00-0.75)$ & 0.707 \\
\hline Enterococcus & $11.60(8.91-18.74)$ & $1.90(1.26-5.90)$ & $0.011^{*}$ \\
\hline Escherichia & $0.44(0.00-2.99)$ & $5.97(0.63-9.91)$ & 0.115 \\
\hline Klebsiella & $0.22(0.00-1.99)$ & $7.22(1.59-22.38)$ & $0.015^{\star}$ \\
\hline Lactobacillus & $0.29(0.08-1.03)$ & $0.68(0.35-0.91)$ & 0.373 \\
\hline Lactococcus & $0.00(0.00-0.16)$ & $0.19(0.08-0.56)$ & $0.044^{\star}$ \\
\hline Parabacteroides & $0.00(0.00-0.13)$ & $0.12(0.07-0.20)$ & 0.120 \\
\hline Rothia & $0.02(0.00-0.07)$ & $0.24(0.11-0.28)$ & $0.017^{\star}$ \\
\hline Staphylococcus & $0.31(0.08-0.59)$ & $0.40(0.04-1.36)$ & 0.765 \\
\hline Streptococcus & $2.57(1.34-6.41)$ & $7.61(3.60-35.26)$ & 0.050 \\
\hline Turicibacter & $0.18(0.05-0.43)$ & $0.30(0.15-0.40)$ & 0.597 \\
\hline Veillonella & $0.03(0.00-0.18)$ & $0.36(0.00-0.84)$ & 0.283 \\
\hline
\end{tabular}

Data are expressed as the medians (IQRS) of the taxa with a median relative abundance of $>0.1 \%$ in at least one group. Intergroup differences were analyzed using the Mann-Whitney $U$-test. ${ }^{*} P<0.05$; ${ }^{* *} P<0.01$. IQRs, interquartile range. 

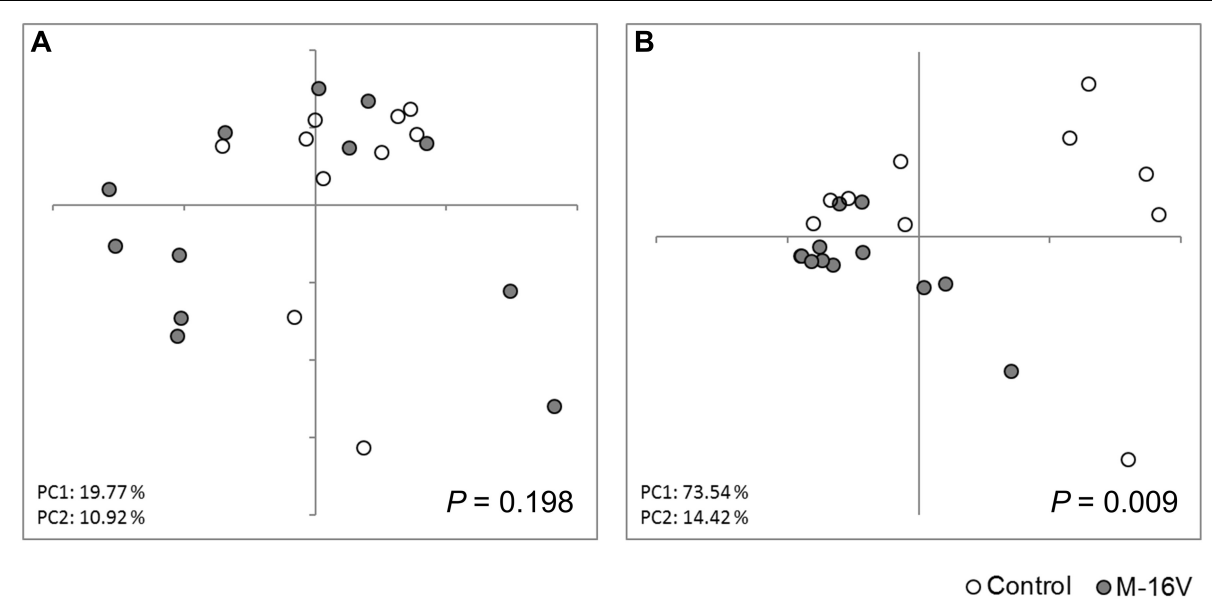

FIGURE 2 | Gut microbiota profiles by PCoA. (A) Unweighted and (B) weighted UniFrac PCoA of the fecal microbiota obtained from subjects in the control group $(n=10)$ and the M-16V group $(n=12)$. Intergroup differences were analyzed using PERMANOVA.

We investigated the influence of factors such as gestational age and birth/discharge weight on the intestinal microbiota. There were significant correlations between the relative abundance of Actinobacteria/Proteobacteria, which were the major differences between the two groups, and some factors including birth weight and others (Supplementary Figures 1, 2). In addition, we found that factors such as gestational age and birth/discharge weight contributed a small proportion of variance, and oral administration of $B$. breve $\mathrm{M}-16 \mathrm{~V}$ was the only significant variable explaining variance in the infant microbiota composition, by the PERMANOVA multivariate analysis using a weighted UniFrac matrix (Table 3).

\section{Quantitative PCR Detection of Bifidobacterium Species}

Because the abundance difference in Bifidobacterium between the groups was the most remarkable, we investigated the bifidobacteria composition at the species level in addition to analyzing the $B$. breve $\mathrm{M}-16 \mathrm{~V}$ abundance by qPCR. Notably, $B$. breve $\mathrm{M}-16 \mathrm{~V}$ was detected in the fecal samples from all subjects in the M-16V group except for two infants (Subject Nos. 9 and 10 in Figure 3), suggesting that this strain colonized a subset of infants for at least several weeks after discontinuing the probiotics. The cell numbers for Bifidobacterium spp., B. breve, the $B$. longum group, and the $B$. catenulatum group were significantly higher in the $\mathrm{M}-16 \mathrm{~V}$ group than in the control group (Figure 4).

\section{DISCUSSION}

Probiotics supplementation is a promising approach to improve dysbiosis and prevent gut microbiota-associated diseases in LBW infants. However, it is unclear whether the microbial components in probiotic treatments can persist in the gut during early life, although some studies indicated the potential for some probiotics
(Frese et al., 2017; Alcon-Giner et al., 2020; Yousuf et al., 2020) as described below. This study found the colonization of $B$. breve M$16 \mathrm{~V}$ in the intestinal tract of most LBW infants for at least several weeks following cessation of its administration as a probiotic. It has been reported that the persistence of introduced probiotics in the adult gut is limited. Most probiotic strains were only detectable for less than two weeks after the administration period despite their high detection rates in the gastrointestinal tract during probiotic treatment (Alander et al., 2001; Frese et al., 2012; Charbonneau et al., 2013). One notable study suggested a possible probiotic colonization in $30 \%$ of the adult subjects for up to 6 months after administration (Maldonado-Gómez et al., 2016). In contrast with adults, whose gut microbiota remains relatively stable (Faith et al., 2013), the composition of the gut microbiota in infants reportedly shows great shifts up to 2-4 years old when it reaches a more stable and mature composition (Voreades et al., 2014; Odamaki et al., 2016; Stewart et al., 2018). Furthermore, the microbiota composition is more immature and less stable in premature infants than full-term infants (Gritz and Bhandari, 2015; Henderickx et al., 2019). This instability of the gut microbiota in addition to the low abundance of Bifidobacterium potentially provided a niche opportunity for oral administration of bifidobacteria probiotic strains such as $B$. breve $\mathrm{M}-16 \mathrm{~V}$ in $\mathrm{LBW}$ infants.

Another reason for the high colonization of $B$. breve $\mathrm{M}-16 \mathrm{~V}$ might arise from the species-specific property. Bifidobacteria display a difference in their ecological adaptation among species and show genotypic and physiological differences related to their different residential origin. Bifidobacterial species of human origin are grouped as human-residential bifidobacteria (HRB) (Odamaki et al., 2015; Wong et al., 2018). Among HRB, B. breve, B. longum subsp. infantis, B. longum subsp. longum, and $B$. bifidum, which are prevalently isolated from the infant's intestine, are referred to as infant-type HRB. Unlike non-HRB such as Bifidobacterium animalis subsp. lactis and Bifidobacterium thermophilum, infant-type HRB have been reported to possess high ability to assimilate human milk 


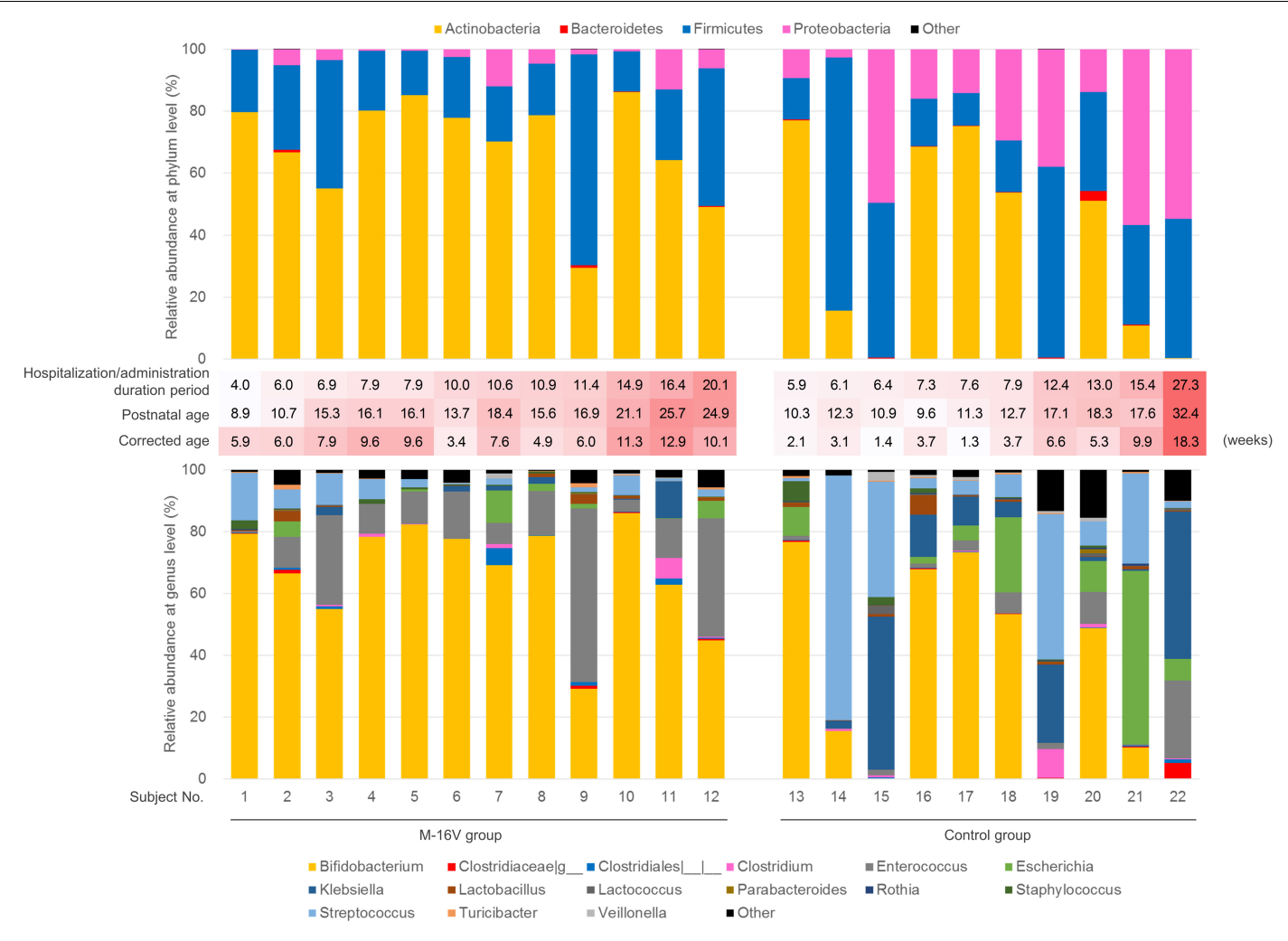

FIGURE 3 | Fecal microbiota composition of each subject. Relative abundance of each phylum (upper) and genus (lower) of microbiota of each subject. The four phyla and 15 genera indicated in Table 2 were shown. In the middle of the figures, hospitalization period (that is, probiotic administration duration in the M-16V group), postnatal and corrected age at the fecal sampling scaled by red color were indicated. The darker the red color, the higher the value of administration duration/hospitalization period, postnatal age, and corrected age, respectively. Subject No. 1-12 were in the M-16V group, and subject No. 13-22 were in the control group.

oligosaccharides (HMOs) and undergo specific adaptation to the infant host (Wong et al., 2018). Indeed, Underwood et al. (2013) showed that B. longum subsp. infantis colonization was better than $B$. animalis subsp. lactis colonization in both formulafed and human milk-fed premature infants. In human milk-fed

TABLE 3 | Multivariate analysis using PERMANOVA to test the variation in microbiota composition explained by each factor.

\begin{tabular}{lcc}
\hline Variable & $\boldsymbol{R}^{\mathbf{2}}$ & $\boldsymbol{P}$-value \\
\hline Treatment (M-16V or Control) & 0.238 & $0.009^{\star \star}$ \\
Gestational age $(<30$ or $\geq 30$ weeks) & 0.035 & 0.459 \\
Birth weight $(<1,200$ or $\geq 1,200 \mathrm{~g})$ & 0.076 & 0.183 \\
Discharge weight $(<3,000$ or $\geq 3,000 \mathrm{~g})$ & 0.036 & 0.444 \\
Hospitalization period $(<10$ or $\geq 10$ weeks $)$ & 0.029 & 0.527 \\
Postnatal age at the fecal sampling ( $<16$ or $\geq 16$ weeks) & 0.026 & 0.565 \\
Corrected age at the fecal sampling $(<7$ or $\geq 7$ weeks) & 0.016 & 0.746 \\
Fecal sampling ( $<5$ or $\geq 5$ weeks after discharge) & 0.019 & 0.692 \\
Residuals & 0.524 & \\
Total & 1.000 & \\
\hline
\end{tabular}

The PERMANOVA multivariate analysis was performed using a weighted UniFrac matrix.

${ }^{* *} P<0.01$. infants, greater increases in fecal Bifidobacterium and decreases in $\gamma$-Proteobacteria followed the administration of B. longum subsp. infantis than that of B. animalis subsp. lactis (Underwood et al., 2013). Another study confirmed that B. breve or B. longum subsp. infantis were early colonizers apparently independent of early life-events, such as mode of delivery and type of feeding, while the colonization of $B$. animalis subsp. lactis was dependent solely on the type of feeding (Martin et al., 2016). The authors suggested that the frequent colonization by $B$. animalis subsp. lactis in infants exposed to formula feeding may result from the use of formula supplemented with probiotic strains belonging to this subspecies. These findings suggest that infant-type HRB are more effective colonizers of the infant gut. Consistent with this finding, it was shown the persistent colonization of probiotic strains of B. longum, B. bifidum, and B. breve up to 5 months after supplementation of commercially probiotics containing these Bifidobacterium strains and Lactobacillus rhamnosus strain in preterm infants (Yousuf et al., 2020). Another study also indicated that the administration of B. longum subsp. infants resulted in a colonization period of at least a month in the breast-fed infant gut (Frese et al., 2017). The authors postulated that the colonization of this strain was attributed to the ancient adaptations of B. longum subsp. infantis to HMOs; that is, the capacity to transport these substances into this bacterium's 

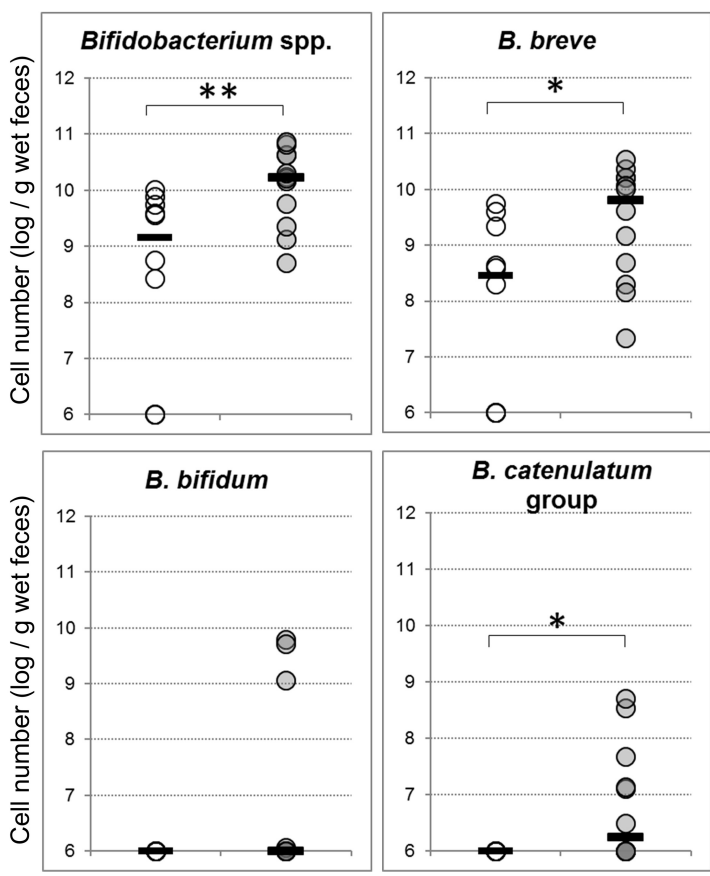
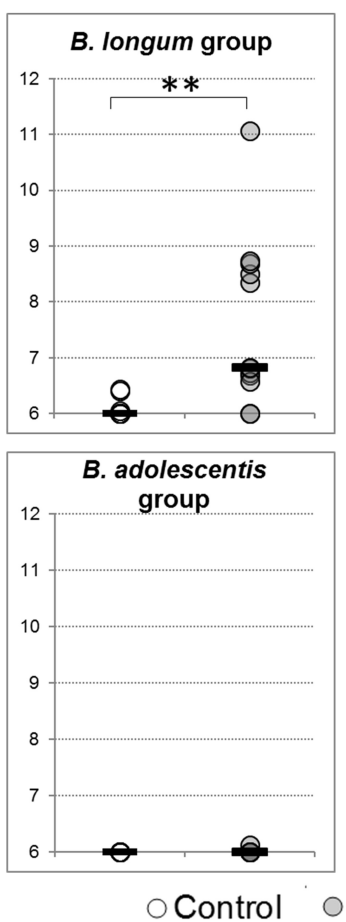

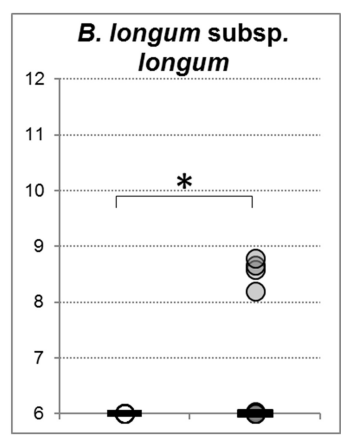

B. breve M-16V

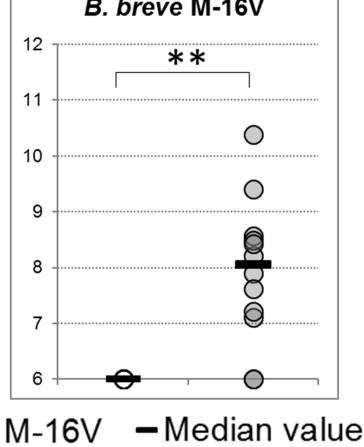

FIGURE 4 | Quantitative PCR detection of Bifidobacterium species and B. breve M-16V. Cell numbers were determined as the log 10 of cells per gram wet weight in each fecal sample. The detection limit was below $10^{6} / \mathrm{g}$ wet weight of feces. Intergroup differences were analyzed using the Mann-Whitney $U$-test. ${ }^{*} P<0.05$; $* * P<0.01$

cytoplasm and consume the full range of HMOs (Underwood et al., 2015). Through their study on probiotic supplementation of preterm infants (Alcon-Giner et al., 2020), suggested the ability of the probiotic B. bifidum strain to colonize the preterm infants' gut by showing its presence in two fecal samples collected at 41 and 50 days after supplementation. Noteworthy, genomic analysis of this strain showed the presence of genes involved in HMO utilization and mucin degradation which may aid the gut persistence (Alcon-Giner et al., 2020). In the present study, $B$. breve $\mathrm{M}-16 \mathrm{~V}$ was detected in $83.3 \%$ of fecal samples from LBW infants in M-16V group at 3.7-9.3 weeks following its cessation of administration. Unfortunately, there were too few subjects to evaluate the influence of the infant feeding methods on the colonization of $B$. breve $\mathrm{M}-16 \mathrm{~V}$ in this study; however, we noted that the two samples from the infants where $B$. breve M-16V was not detected were mixed-fed with breast milk and infant formula (data not shown). Further studies are needed to investigate the environmental factors that could affect the colonization of probiotics (e.g., whether or not the maternal genetic background related to HMO secretion or the feeding method affects colonization).

Recent studies have shown that the introduction of live microbes does not result in significant alterations of the fecal microbiota in healthy adults (Kim et al., 2013; Kristensen et al., 2016). Studies on infants are controversial with respect to this finding. Ishizeki et al. (2013) reported that the supplementation of single ( $B$. breve $\mathrm{M}-16 \mathrm{~V}$ ) or multiple (B. breve $\mathrm{M}-16 \mathrm{~V}$, $B$. longum subsp. infantis M-63, and B. longum subsp. longum
BB536) infant-type HRB strains to LBW infants resulted in the increase of the detection rate and number of Bifidobacterium in feces. Also, a study by Plummer et al. (2018) indicated that probiotic supplementation with $B$. longum subsp. infantis BB02, Streptococcus thermophilus $\mathrm{TH}-4$ and $B$. animalis subsp. lactis BB-12 from soon after birth increased the abundance of Bifidobacterium in the gut microbiota of very preterm infants during supplementation period. Furthermore, potential longterm contribution of probiotic strains on development of gut microbiota in preterm infants have been indicated by some researches (Alcon-Giner et al., 2020; Yousuf et al., 2020). On the other hand, a double-blind, randomized placebo-controlled intervention showed that intake of Lactobacillus acidophilus NCFM or B. animalis subsp. lactis Bi-07 to young children with atopic dermatitis for eight weeks did not affect the composition and diversity of the main bacterial populations in feces (Larsen et al., 2011). Similarly, no effect on the overall microbiota composition was observed when Lactobacillus reuteri DSM 17938 was administrated to breast-fed colicky infants for 21 days (Roos et al., 2013). We found that B. breve M-16V administration significantly impacted the overall microbiota composition beyond the non-administration period of 3.7-9.3 weeks. In the M-16V group, the relative abundance of Proteobacteria was significantly lower than in the control group. A sustained increase in Proteobacteria abundance is considered a signature of dysbiosis (Shin et al., 2015). Some reports have indicated an association between intestinal Proteobacteria and NEC in premature infants (Wang et al., 2009; Pammi et al., 
2017; Lindberg et al., 2020). Mirpuri et al. (2014) found that the IgA-dependent suppression of Proteobacteria in the infant gut was important for establishing a beneficial commensal population and reducing susceptibility to colonic injury and inflammation. At the genus level, the relative abundance of Klebsiella, which have been associated with neonatal bacterial infections (Podschun and Ullmann, 1998; Hornik et al., 2012) and NEC (Sim et al., 2015; Olm et al., 2019), were lower in the $\mathrm{M}-16 \mathrm{~V}$ group than in the control group. Contrastingly, the relative abundance of Bifidobacterium was significantly higher in the $\mathrm{M}-16 \mathrm{~V}$ group than in the control group. It has been reported that a higher abundance of Bifidobacterium in early infancy is associated with better immune system responses to vaccination, potentially enhancing immunological memory (Huda et al., 2019). Conversely, a lower abundance of Bifidobacteriaceae, which primarily includes the Bifidobacterium genus, is suggested to trigger the development of allergic sensitization, eczema, or asthma (Zimmermann et al., 2019).

Factors such as gestational age and birth weight have been reported to affect the developing gut microbiota in preterm neonates (Korpela et al., 2018; Henderickx et al., 2019; Alcon-Giner et al., 2020). Some factors including birth weight had significant correlations with the relative abundance of Actinobacteria/Proteobacteria. A part of these correlations might be due to the significant difference of the birth body weight between groups, and the close association among the birth weight, the gestational age, and the hospitalization period (Supplementary Figure 3). Our PERMANOVA multivariate analysis confirmed that the effect of the supplementation of B. breve $\mathrm{M}-16 \mathrm{~V}$ on the gut microbiota was greater than that of other factors such as gestational age and birth weight.

Our qPCR analysis revealed that the cell numbers of the administrated strain and some of the indigenous bifidobacteria species were significantly higher in the M-16V group. It remains unclear as to why administering $B$. breve $\mathrm{M}-16 \mathrm{~V}$ promoted the colonization of other bifidobacteria, but one possibility is that because acetic acid is the main metabolite it might suppress the growth of acid-sensitive bacteria such as Proteobacteria, thereby providing an appropriate environment for bifidobacteria growth. Overall, our findings suggest that $B$. breve $\mathrm{M}-16 \mathrm{~V}$ administration can contribute to the establishment of a healthy gut microbiota composition in LBW infants.

There are several limitations in this study. First, this study is not a randomized controlled trial and included a small number of infants. Second, the birth weight and hospital discharge weight were significantly higher in the $\mathrm{M}-16 \mathrm{~V}$ group than in the control group. Body weight is an important indicator of infant maturity and a key factor influencing the intestinal microbiota in neonates, that cannot be ignored especially in LBW infants. Third, the period from the discharge, that is, the cessation of probiotic administration in the $\mathrm{M}-16 \mathrm{~V}$ group to the fecal sampling was significantly longer in the M-16V group than the control group, though the postnatal and the corrected age at sampling were not significantly different between the groups. Since the composition of the gut microbiota dramatically changes in the early life stage, the difference of the period from the hospital discharge to the fecal sampling could lead to the difference in the gut microbiota composition. However, it is assumed that the longer non-administration period in the M-16V group would not bring the overestimation of the colonization of $B$. breve $\mathrm{M}-16 \mathrm{~V}$ in the infant's gut. Fourth, subjects in the two groups were from different hospitals, and the inclusion period lasted over 5 years. Such a long period might lead to change the hospital environment. The establishment of gut microbiota in very preterm infants is unstable and susceptible to the environment factors (Brooks et al., 2014) and the NICU practices (Rozé et al., 2020). Hence, a large-scale, double-blind, placebo-controlled study in the matched infants in the same facility with sequential fecal sampling from birth will be necessary to confirm the beneficial effects of $B$. breve $\mathrm{M}-16 \mathrm{~V}$ that have been suggested in this study.

In conclusion, our results show that oral administration of $B$. breve $\mathrm{M}-16 \mathrm{~V}$ leads to its colonization in the infant gut for at least several weeks after administration and potentially contributes to improved gut microbiota establishment. Further follow-up investigations will help to elucidate the durability of these effects through later childhood, and whether these effects carry implications for overall health later in life.

\section{DATA AVAILABILITY STATEMENT}

The datasets presented in this study can be found in online repositories. The names of the repository/repositories and accession number(s) can be found below: https://www.ddbj.nig. ac.jp/, DRA010463.

\section{ETHICS STATEMENT}

The studies involving human participants were reviewed and approved by the Ethics Committee of Juntendo University Hospital. Written informed consent to participate in this study was provided by the participants' legal guardian/next of kin.

\section{AUTHOR CONTRIBUTIONS}

J-ZX and TS conceived and designed the study. AH, NI, and $\mathrm{KH}$ performed the clinical trial and experiments. $\mathrm{AH}$ and $\mathrm{TO}$ analyzed the data and wrote the manuscript. TS supervised the overall study. All authors have read and approved the final manuscript.

\section{ACKNOWLEDGMENTS}

We thank Sachiko Takahashi, Eri Mitsuyama, and Nanami Hashikura for their technical support. We also thank Sandra Cheesman from Edanz Group (https://en-authorservices.edanzgroup.com/ac) for editing a draft of this manuscript. 


\section{SUPPLEMENTARY MATERIAL}

The Supplementary Material for this article can be found online at: https://www.frontiersin.org/articles/10.3389/fmicb. 2021.610080/full\#supplementary-material

Supplementary Figure 1 | Relationship between Actinobacteria and subject's characteristics. Relationships between the relative abundance of Actinobacteria and the gestational age (A), the birth weight (B), the discharge weight (C), the hospitalization/probiotic administration period (D), the postnatal age at the fecal sampling (E), the corrected age at the fecal sampling (F), or the fecal sampling timing (G) in all subjects (black dashed line), the M-16V group (red circles and line), or the control group (blue circles and line). Associations were assessed by Spearman's rank correlation test. ${ }^{*} P<0.05$.

Supplementary Figure 2 | Relationship between Proteobacteria and subject's characteristics. Relationships between the relative abundance of Proteobacteria

\section{REFERENCES}

Alander, M., Mättö, J., Kneifel, W., Johansson, M., Kögler, B., Crittenden, R., et al. (2001). Effect of galacto-oligosaccharide supplementation on human faecal microflora and on survival and persistence of Bifidobacterium lactis Bb-12 in the gastrointestinal tract. Int. Dairy J. 11, 817-825. doi: 10.1016/S0958-6946(01) 00100- 5

Alcon-Giner, C., Dalby, M. J., Caim, S., Ketskemety, J., Shaw, A., Sim, K., et al. (2020). Microbiota supplementation with Bifidobacterium and Lactobacillus modifies the preterm infant gut microbiota and metabolome: an observational study. Cell Rep. Med. 1:100077. doi: 10.1016/j.xcrm.2020.10 0077

Arrieta, M. C., Arévalo, A., Stiemsma, L., Dimitriu, P., Chico, M. E., Loor, S., et al. (2018). Associations between infant fungal and bacterial dysbiosis and childhood atopic wheeze in a nonindustrialized setting. J. Allergy Clin. Immunol. 142, 424-434. doi: 10.1016/j.jaci.2017.08.041

Arrieta, M.-C., Stiemsma, L. T., Dimitriu, P. A., Thorson, L., Russell, S., YuristDoutsch, S., et al. (2015). Early infancy microbial and metabolic alterations affect risk of childhood asthma. Sci. Transl. Med. 7:307ra152. doi: 10.1126/ scitranslmed.aab2271

Athalye-Jape, G., Rao, S., Simmer, K., and Patole, S. (2018). Bifidobacterium breve M-16V as a probiotic for preterm infants: a strain-specific systematic review. J. Parenter. Enter. Nutr. 42, 677-688. doi: 10.1177/014860711772 2749

Brooks, B., Firek, B. A., Miller, C. S., Sharon, I., Thomas, B. C., Baker, R., et al. (2014). Microbes in the neonatal intensive care unit resemble those found in the gut of premature infants. Microbiome 2:1. doi: 10.1186/2049-2618-2-1

Callahan, B. J., McMurdie, P. J., Rosen, M. J., Han, A. W., Johnson, A. J. A., and Holmes, S. P. (2016). DADA2: high-resolution sample inference from Illumina amplicon data. Nat. Methods 13, 581-583. doi: 10.1038/nmeth.3869

Charbonneau, D., Gibb, R. D., and Quigley, E. M. M. (2013). Fecal excretion of Bifidobacterium infantis 35624 and changes in fecal microbiota after eight weeks of oral supplementation with encapsulated probiotic. Gut Microbes 4, 201-211. doi: 10.4161/gmic.24196

Cox, L. M., Yamanishi, S., Sohn, J., Alekseyenko, A. V., Leung, M., Cho, I., et al. (2014). Altering the intestinal microbiota during a critical developmental window has lasting metabolic consequences. Cell 158, 705-721. doi: 10.1016/j. cell.2014.05.052

Dalby, M. J., and Hall, L. J. (2020). Recent advances in understanding the neonatal microbiome. F1000Research 9:422. doi: 10.12688/f1000research.22355.1

Di Gioia, D., Aloisio, I., Mazzola, G., and Biavati, B. (2014). Bifidobacteria: their impact on gut microbiota composition and their applications as probiotics in infants. Appl. Microbiol. Biotechnol. 98, 563-577. doi: 10.1007/s00253-0135405-9

Faith, J. J., Guruge, J. L., Charbonneau, M., Subramanian, S., Seedorf, H., Goodman, A. L., et al. (2013). The long-term stability of the human gut microbiota. Science 341:1237439. doi: 10.1126/science.1237439 and the gestational age (A), the birth weight (B), the discharge weight $(\mathbf{C})$, the hospitalization/probiotic administration period (D), the postnatal age at the fecal sampling (E), the corrected age at the fecal sampling (F), or the fecal sampling timing (G) in all subjects (black dashed line), the M-16V group (red circles and line), or the control group (blue circles and line). Associations were assessed by Spearman's rank correlation test. ${ }^{\star} P<0.05 ;{ }^{\star \star} P<0.01$.

Supplementary Figure $\mathbf{3}$ | Relationship between the birth weight, gestational age, and hospitalization. Relationships between the birth weight and gestational age (A), the birth weight and the hospitalization period (B), and the gestational age and the hospitalization period (C) in all subjects (black dashed line), the M-16V group (red circles and line), or the control group (blue circles and line). Associations were assessed by Spearman's rank correlation test. ${ }^{\star} P<0.05$; ${ }^{\star \star} P<0.01$.

Supplementary Table 1 | Study schedule for each subject.

Supplementary Table 2 | Quantitative PCR primer sets.

Supplementary Table 3 | Alpha-diversity of the gut microbiota.

Frese, S. A., Hutkins, R., and Walter, J. (2012). Comparison of the colonization ability of autochthonous and allochthonous strains of lactobacilli in the human gastrointestinal tract. Adv. Microbiol. 2, 399-409. doi: 10.4236/aim.2012. 23051

Frese, S. A., Hutton, A. A., Contreras, L. N., Shaw, C. A., Palumbo, M. C., Casaburi, G., et al. (2017). Persistence of supplemented Bifidobacterium longum subsp. infantis EVC001 in breastfed infants. mSphere 2, e501-e517. doi: 10.1128/ mSphere.00501-17

Gritz, E. C., and Bhandari, V. (2015). The human neonatal gut microbiome: a brief review. Front. Pediatr. 3:17. doi: 10.3389/fped.2015.00017

Henderickx, J. G. E., Zwittink, R. D., van Lingen, R. A., Knol, J., and Belzer, C. (2019). The preterm gut microbiota: an inconspicuous challenge in nutritional neonatal care. Front. Cell. Infect. Microbiol. 9:85. doi: 10.3389/fcimb.2019. 00085

Hornik, C. P., Fort, P., Clark, R. H., Watt, K., Benjamin, D. K., Smith, P. B., et al. (2012). Early and late onset sepsis in very-low-birth-weight infants from a large group of neonatal intensive care units. Early Hum. Dev. 88, S69-S74. doi: 10.1016/S0378-3782(12)70019-1

Huda, M. N., Ahmad, S. M., Alam, M. J., Khanam, A., Kalanetra, K. M., Taft, D. H., et al. (2019). Bifidobacterium abundance in early infancy and vaccine response at 2 years of age. Pediatrics 143:e20181489. doi: 10.1542/peds.2018-1489

Ishizeki, S., Sugita, M., Takata, M., and Yaeshima, T. (2013). Effect of administration of bifidobacteria on intestinal microbiota in low-birth-weight infants and transition of administered bifidobacteria: a comparison between one-species and three-species administration. Anaerobe 23, 38-44. doi: 10.1016/ j.anaerobe.2013.08.002

Kanda, Y. (2013). Investigation of the freely available easy-to-use software "EZR" for medical statistics. Bone Marrow Transplant. 48, 452-458. doi: 10.1038/bmt. 2012.244

Kato, K., Odamaki, T., Mitsuyama, E., Sugahara, H., Xiao, J. Z., and Osawa, R. (2017). Age-related changes in the composition of gut Bifidobacterium species. Curr. Microbiol. 74, 987-995. doi: 10.1007/s00284-017-1272-4

Kim, S.-W., Suda, W., Kim, S., Oshima, K., Fukuda, S., Ohno, H., et al. (2013). Robustness of gut microbiota of healthy adults in response to probiotic intervention revealed by high-throughput pyrosequencing. DNA Res. 20, $241-$ 253. doi: 10.1093/dnares/dst006

Korpela, K., Blakstad, E. W., Moltu, S. J., Strømmen, K., Nakstad, B., Rønnestad, A. E., et al. (2018). Intestinal microbiota development and gestational age in preterm neonates. Sci. Rep. 8:2453. doi: 10.1038/s41598-018-20827-x

Kostic, A. D., Gevers, D., Siljander, H., Vatanen, T., Peet, A., Tillmann, V., et al. (2015). The dynamics of the human infant gut microbiome in development and in progression towards type 1 diabetes. Cell Host Microbe 17, 260-273. doi: 10.1016/j.chom.2015.01.001

Kristensen, N. B., Bryrup, T., Allin, K. H., Nielsen, T., Hansen, T. H., and Pedersen, O. (2016). Alterations in fecal microbiota composition by probiotic supplementation in healthy adults: a systematic review of randomized controlled trials. Genome Med. 8:52. doi: 10.1186/s13073-016-0300-5 
Larsen, N., Vogensen, F. K., Gøbel, R., Michaelsen, K. F., Abu Al-Soud, W., Sørensen, S. J., et al. (2011). Predominant genera of fecal microbiota in children with atopic dermatitis are not altered by intake of probiotic bacteria Lactobacillus acidophilus NCFM and Bifidobacterium animalis subsp. lactis Bi-07. FEMS Microbiol. Ecol. 75, 482-496. doi: 10.1111/j.1574-6941.2010. 01024.x

Leahy, S. C., Higgins, D. G., Fitzgerald, G. F., and van Sinderen, D. (2005). Getting better with bifidobacteria. J. Appl. Microbiol. 98, 1303-1315. doi: 10.1111/j. 1365-2672.2005.02600.x

Lindberg, T. P., Caimano, M. J., Hagadorn, J. I., Bennett, E. M., Maas, K., Brownell, E. A., et al. (2020). Preterm infant gut microbial patterns related to the development of necrotizing enterocolitis. J. Matern. Neonatal Med. 33, 349-358. doi: 10.1080/14767058.2018.1490719

Maldonado-Gómez, M. X., Martínez, I., Bottacini, F., O’Callaghan, A., Ventura, M., van Sinderen, D., et al. (2016). Stable engraftment of Bifidobacterium longum AH1206 in the human gut depends on individualized features of the resident microbiome. Cell Host Microbe 20, 515-526. doi: 10.1016/j.chom.2016. 09.001

Martin, R., Makino, H., Yavuz, A. C., Ben-Amor, K., Roelofs, M., Ishikawa, E., et al. (2016). Early-life events, including mode of delivery and type of feeding, siblings and gender, shape the developing gut microbiota. PLoS One 11:e0158498. doi: 10.1371/journal.pone.0158498

Matsuki, T., Watanabe, K., Fujimoto, J., Kado, Y., Takada, T., Matsumoto, K., et al. (2004). Quantitative PCR with 16 S rRNA-gene-targeted speciesspecific primers for analysis of human intestinal bifidobacteria. Appl. Environ. Microbiol. 70, 167-173. doi: 10.1128/aem.70.1.167

Matsuki, T., Watanabe, K., Tanaka, R., and Oyaizu, H. (1998). Rapid identification of human intestinal bifidobacteria by $16 \mathrm{~S}$ rRNA-targeted species- and groupspecific primers. FEMS Microbiol. Lett. 167, 113-121. doi: 10.1111/j.1574-6968. 1998.tb13216.x

Mirpuri, J., Raetz, M., Sturge, C. R., Wilhelm, C. L., Benson, A., Savani, R. C., et al. (2014). Proteobacteria-specific IgA regulates maturation of the intestinal microbiota. Gut Microbes 5, 28-39. doi: 10.4161/gmic.26489

Mohan, R., Koebnick, C., Schildt, J., Schmidt, S., Mueller, M., Possner, M., et al. (2006). Effects of Bifidobacterium lactis Bb12 supplementation on intestinal microbiota of preterm infants: a double-blind, placebo-controlled, randomized study. J. Clin. Microbiol. 44, 4025-4031. doi: 10.1128/JCM.00 767-06

Neu, J., and Pammi, M. (2017). Pathogenesis of NEC: impact of an altered intestinal microbiome. Semin. Perinatol. 41, 29-35. doi: 10.1053/j.semperi.2016. 09.015

Odamaki, T., Horigome, A., Sugahara, H., Hashikura, N., Minami, J., Xiao, J., et al. (2015). Comparative genomics revealed genetic diversity and species/strain-level differences in carbohydrate metabolism of three probiotic bifidobacterial species. Int. J. Genomics 2015:567809. doi: 10.1155/2015/56 7809

Odamaki, T., Kato, K., Sugahara, H., Hashikura, N., Takahashi, S., Xiao, J., et al. (2016). Age-related changes in gut microbiota composition from newborn to centenarian: a cross-sectional study. BMC Microbiol. 16:90. doi: 10.1186/ s12866-016-0708-5

Olm, M. R., Bhattacharya, N., Crits-Christoph, A., Firek, B. A., Baker, R., Song, Y. S., et al. (2019). Necrotizing enterocolitis is preceded by increased gut bacterial replication, Klebsiella, and fimbriae-encoding bacteria. Sci. $A d v$. 5:eaax5727. doi: 10.1126/sciadv.aax5727

Pammi, M., Cope, J., Tarr, P. I., Warner, B. B., Morrow, A. L., Mai, V., et al. (2017). Intestinal dysbiosis in preterm infants preceding necrotizing enterocolitis: a systematic review and meta-analysis. Microbiome 5:31. doi: 10.1186/s40168017-0248-8

Patel, A. L., Mutlu, E. A., Sun, Y., Koenig, L., Green, S., Jakubowicz, A., et al. (2016). Longitudinal survey of microbiota in hospitalized preterm very-lowbirth-weight infants. J. Pediatr. Gastroenterol. Nutr. 62, 292-303. doi: 10.1097/ MPG.0000000000000913

Patole, S. K., Rao, S. C., Keil, A. D., Nathan, E. A., Doherty, D. A., and Simmer, K. N. (2016). Benefits of Bifidobacterium breve M-16V supplementation in preterm neonates - a retrospective cohort study. PLoS One 11:e0150775. doi: 10.1371/journal.pone.0150775
Patole, S., Keil, A., Chang, A., Nathan, E., Doherty, D., Simmer, K., et al. (2014). Effect of Bifidobacterium breve M-16V supplementation on fecal bifidobacteria in preterm neonates - a randomised double blind placebo controlled trial. PLoS One 9:e89511. doi: 10.1371/journal.pone.008 9511

Plummer, E. L., Bulach, D. M., Murray, G. L., Jacobs, S. E., Tabrizi, S. N., Garland, S. M., et al. (2018). Gut microbiota of preterm infants supplemented with probiotics: sub-study of the ProPrems trial. BMC Microbiol. 18:184. doi: 10 . 1186/s12866-018-1326-1

Podschun, R., and Ullmann, U. (1998). Klebsiella spp. as nosocomial pathogens: epidemiology, taxonomy, typing methods, and pathogenicity factors. Clin. Microbiol. Rev. 11, 589-603.

Roos, S., Dicksved, J., Tarasco, V., Locatelli, E., Ricceri, F., Grandin, U., et al. (2013). 454 pyrosequencing analysis on faecal samples from a randomized DBPC trial of colicky infants treated with Lactobacillus reuteri DSM 17938. PLoS One 8:e56710. doi: 10.1371/journal.pone.0056710

Rozé, J.-C., Ancel, P.-Y., Marchand-Martin, L., Rousseau, C., Montassier, E., Monot, C., et al. (2020). Assessment of neonatal intensive care unit practices and preterm newborn gut microbiota and 2-year neurodevelopmental outcomes. JAMA Netw. Open 3:e2018119. doi: 10.1001/jamanetworkopen.2020. 18119

Satoh, T., Izumi, H., Iwabuchi, N., Odamaki, T., Namba, K., Abe, F., et al. (2016). Bifidobacterium breve prevents necrotising enterocolitis by suppressing inflammatory responses in a preterm rat model. Benef. Microbes 7, 75-82. doi: 10.3920/BM2015.0035

Shin, N. R., Whon, T. W., and Bae, J. W. (2015). Proteobacteria: microbial signature of dysbiosis in gut microbiota. Trends Biotechnol. 33, 496-503. doi: 10.1016/j. tibtech.2015.06.011

Sim, K., Shaw, A. G., Randell, P., Cox, M. J., McClure, Z. E., Li, M.-S., et al. (2015). Dysbiosis anticipating necrotizing enterocolitis in very premature infants. Clin. Infect. Dis. 60, 389-397. doi: 10.1093/cid/ciu822

Stewart, C. J., Ajami, N. J., O’Brien, J. L., Hutchinson, D. S., Smith, D. P., Wong, M. C., et al. (2018). Temporal development of the gut microbiome in early childhood from the TEDDY study. Nature 562, 583-588. doi: 10.1038/s41586018-0617-x

Stewart, C. J., Embleton, N. D., Marrs, E. C. L., Smith, D. P., Nelson, A., Abdulkadir, B., et al. (2016). Temporal bacterial and metabolic development of the preterm gut reveals specific signatures in health and disease. Microbiome 4:67. doi: 10.1186/s40168-016-0216-8

Sugahara, H., Odamaki, T., Hashikura, N., Abe, F., and Xiao, J. (2015). Differences in folate production by bifidobacteria of different origins. Biosci. Microbiota Food Health 34, 87-93. doi: 10.12938/bmfh.2015003

Umezaki, H., Shinohara, K., Satoh, Y., Shoji, H., Satoh, H., Ohtsuka, Y., et al. (2010). Bifidobacteria prevents preterm infants from developing infection and sepsis. Int. J. Probiotics Prebiotics 5, 33-36.

Underwood, M. A., German, J. B., Lebrilla, C. B., and Mills, D. A. (2015). Bifidobacterium longum subspecies infantis: champion colonizer of the infant gut. Pediatr. Res. 77, 229-235. doi: 10.1038/pr.2014.156

Underwood, M. A., Kalanetra, K. M., Bokulich, N. A., Lewis, Z. T., Mirmiran, M., Tancredi, D. J., et al. (2013). A comparison of two probiotic strains of bifidobacteria in premature infants. J. Pediatr. 163, 1585-1591.e9. doi: 10.1016/ j.jpeds.2013.07.017

Untergasser, A., Cutcutache, I., Koressaar, T., Ye, J., Faircloth, B. C., Remm, M., et al. (2012). Primer3-new capabilities and interfaces. Nucleic Acids Res. 40:e115. doi: 10.1093/nar/gks596

Voreades, N., Kozil, A., and Weir, T. L. (2014). Diet and the development of the human intestinal microbiome. Front. Microbiol. 5:494. doi: 10.3389/fmicb.2014. 00494

Wang, Y., Hoenig, J. D., Malin, K. J., Qamar, S., Petrof, E. O., Sun, J., et al. (2009). $16 \mathrm{~S}$ rRNA gene-based analysis of fecal microbiota from preterm infants with and without necrotizing enterocolitis. ISME J. 3, 944-954. doi: 10.1038/ismej. 2009.37

Wong, C. B., Iwabuchi, N., and Xiao, J. Z. (2019). Exploring the science behind Bifidobacterium breve M-16V in infant health. Nutrients 11:1724. doi: 10.3390/ nu11081724 
Wong, C. B., Sugahara, H., Odamaki, T., and Xiao, J. Z. (2018). Different physiological properties of human-residential and non-human-residential bifidobacteria in human health. Benef. Microbes 9, 111-122. doi: 10.3920/ BM2017.0031

Yousuf, E. I., Carvalho, M., Dizzell, S. E., Kim, S., Gunn, E., Twiss, J., et al. (2020). Persistence of suspected probiotic organisms in preterm infant gut microbiota weeks after probiotic supplementation in the NICU. Front. Microbiol. 11:574137. doi: 10.3389/fmicb.2020.57 4137

Zimmermann, P., Messina, N., Mohn, W. W., Finlay, B. B., and Curtis, N. (2019). Association between the intestinal microbiota and allergic sensitization, eczema, and asthma: a systematic review. J. Allergy Clin. Immunol. 143, 467-485. doi: 10.1016/j.jaci.2018. 09.025
Conflict of Interest: AH, TO, NI, and J-ZX were employees of Morinaga Milk Industry Co., Ltd.

The remaining authors declare that the research was conducted in the absence of any commercial or financial relationships that could be construed as a potential conflict of interest.

Copyright $\odot 2021$ Horigome, Hisata, Odamaki, Iwabuchi, Xiao and Shimizu. This is an open-access article distributed under the terms of the Creative Commons Attribution License (CC BY). The use, distribution or reproduction in other forums is permitted, provided the original author(s) and the copyright owner(s) are credited and that the original publication in this journal is cited, in accordance with accepted academic practice. No use, distribution or reproduction is permitted which does not comply with these terms. 